\title{
An NMR Study of Solvent Interactions in a Paramagnetic System
}

\author{
R. M. Golding, * R. O. Pascual, C. Suvanprakorn, and I. G. Dance
}

\author{
School of Chemistry, The University of New Sotth Wales, Sydney, New South Wales 2052, Australia \\ "E-mail: raygolding@im141. aone.net.au \\ Received Augtist 17, 2006
}

\begin{abstract}
This study explores and interprets in a new way the complex solvent and the temperature dependence of the NMR shifts for the $\mathrm{N}-\mathrm{CH}_{2}$ protons in tris $(N, N$-diethyldithiocarbamato) iron(III) in acetone, benzene, carbon disulfide, chloroform, dimethylformamide and pyridine. The NMR shifts are interpreted in terms of the Fermi contact interaction and the dipolar term from the multipole expansion of the interaction of the electron orbital angular momentum and the electron spin dipolar-nuclear spin angular momentum. This analysis yields a direct measure of the effect of the solvent system on the environment of the transition metal ion. The results are analysed in terms of the crystal field environment of the transition metal ion with contributions from (a) the dithiocarbamate ligand (b) the solvent molecules and (c) the interaction of the effective dipole moment of the polar solvent molecule with the transition metal ion complex.
\end{abstract}

Key Words : NMR paramagnetic chemical shifts, Tris( $N, N$-diethyldithiocarbamato) iron(III), Electronnuclear interactions, Solvent-solute interactions

\section{Introduction}

Several years ago we reported' that the reduction potential, $\mathrm{E}_{\mathrm{l} / 2}$, for several iron(III) dithiocarbamates in acetonewater mixtures was markedly dependent with solvent variations at least as great as 0.3 volts between the $\mathrm{E}_{1 / 2}$ values for an iron(III) dithiocarbamate in acetone and in water. We interpreted these results by considering in a simple way, the interaction of a polar molecule in the solvent with the iron(III) dithiocarbamate.

It is known also that the NMR spectra of these compounds are not only markedly temperature dependent ${ }^{2-5}$ but also at least some are solvent dependent. ${ }^{6}$ For example, the magnitude of the NMR shift for the $\mathrm{N}-\mathrm{CH}_{2}$ protons in $\operatorname{tris}(N, N$ diethyldithiocarbamato) iron(III) increases with increasing temperature ${ }^{2}$ when the compound is in a chloroform solution whereas the magnitude of the NMR shift decreases with increasing temperature when the compound is in a dimethylformamide solution.

In this paper we shall extend our earlier interpretation of the redox results to the NMR data for the $\mathrm{N}-\mathrm{CH}_{2}$ protons in $\operatorname{tris}(N, N$-diethyldithiocarbamato) iron(III). We shall show that the solvent dependence of the nmr shifts can be interpreted as arising from solvent interactions with the iron(III) dithiocarbamate system. Although the solvent interactions are small compared with the electronic interactions within the transition metal iron complex the effect is marked. This arises since in these cases for the $\mathrm{d}^{5}$ iron system the ground electronic state is one of two states where the energy separation is sensitive to small changes in the crystal field environment of the transition metal ion.

\section{Theory}

To gain a detailed and an in-depth insight into the paramagnetic shift we would need to know the electronic and physical structure of the iron(III) dithiocarbamate system in a particular solvent. From that information we would be able to calculate exactly the expected paramagnetic shift arising from the exact electron-nuclear interaction. As we do not have such information we need to address the problem in a more simplistic way. Fortunately, as we shall show, the manner in which we shall explore the problem will result in a very good approximation. This aspect will be reinforced later.

Initially, we shall outline the interactions we need to consider for a single d-electron in a crystal field of octahedral symmetry where, in our case, the potential arises dominantly from the dithiocarbamate ligand bonded to the $\mathrm{Fe}^{3+}$ ion and by the solvent in a more minor way. The crystal field potential may be expressed as

$$
\begin{aligned}
\mathrm{V}(\mathbf{r})= & \left.\frac{2}{5}(21 \pi)\right)^{1 / 2}\left\{\frac{\sqrt{7}}{2 \sqrt{3}} \mathrm{Y}_{40}(\theta, \phi)+\frac{\sqrt{5}}{2 \sqrt{6}} \mathrm{Y}_{44}(\theta, \phi)\right. \\
& \left.+\frac{\sqrt{5}}{2 \sqrt{6}} \mathrm{Y}_{4-4}(\theta, \phi)\right\} \Delta
\end{aligned}
$$

From equation ( 1 ) it can be shown that the five d-orbitals are split into two degenerate orbitals designated as e with energy $3 \Delta / 5$ and three degenerate orbitals designated as $t_{2}$ with energy $-2 \Delta / 5$. The separation of the two energy levels is $\Delta$. The crystal field parameter, $\Delta$, may be up to about 50,000 $\mathrm{cm}^{-1}$. For the $\mathrm{d}^{5}$ system we need to consider the crystal field potential and the Coulomb interaction giving rise to a complex energy pattem. When $\Delta$ is about $27,500 \mathrm{~cm}$ we have an example of what is known as an intermediate crystal field case where the ground state, the lowest energy level, may be the energy level designated ${ }^{2} T_{2}$ arising from the $t_{2}{ }^{5}$ electronic configuration or the energy level designated ${ }^{6} \mathrm{~A}_{1}$ 
arising from the $\mathrm{t}_{2}^{3} \mathrm{e}^{2}$ electronic configuration. We may express the energy difference between these two energy levels as

$$
{ }^{6} \mathrm{~A}_{1}-{ }^{2} \mathrm{~T}_{2}=2 \Delta-15 \mathrm{~B}-10 \mathrm{C} \text {. }
$$

The parameters $B$ and $C$ are the usual Racah parameters ${ }^{1+}$ measuring the Coulomb repulsion interaction. The Racah parameters for the $\mathrm{Fe}^{3+}$ ion are about $1075 \mathrm{~cm}^{-1}$ and 3900 $\mathrm{cm}^{-1}$ for the $\mathrm{B}$ and $\mathrm{C}$ respectively. We shall use the approximation $\mathrm{B}=\mathrm{C} / 4=1000 \mathrm{~cm}^{-1}$.

Such an environment applies for the $d^{j}$ system in the series of iron(III) dithiocarbamate complexes. Hence when $\Delta>$ $27,500 \mathrm{~cm}^{-1}$ the ground state is the ${ }^{2} \mathrm{~T}_{2}$ level whereas when $\Delta$ $<27,500 \mathrm{~cm}^{-1}$ the ground state is the ${ }^{6} \mathrm{~A}_{1}$ level.

Furthermore, the crystal field may have a small trigonal component and we need to consider spin orbit coupling within the ${ }^{3} \mathbf{T}_{2}$ level. We shall define the direction of the trigonal distortion as the z-axis. To simplify the calculations we may treat the ${ }^{2} \mathrm{~T}_{2}: \mathrm{t}_{2}{ }^{5}$ level as corresponding to a ${ }^{3} \mathrm{~T}_{2}: \mathrm{t}_{2}$ level where the trigonal and spin orbit coupling interactions are given by

$$
H=-\zeta l . s-\frac{14 \sqrt{\pi}}{3 \sqrt{5}} \delta \mathrm{Y}_{20}(\theta, \phi)
$$

In equation (3) $\zeta$ is the spin orbit coupling constant, which we shall choose as $400 \mathrm{~cm}^{-1}$, and $\delta$ is a measure of the trigonal distortion. The distortion splits the three $t_{2}$ orbitals by $\delta$ with two $\mathrm{t}_{2}$ orbitals at $-\delta / 3$ and the other $\mathrm{t}_{2}$ orbital at $2 \delta /$ 3.

From the NMR results of the temperature dependence of the proton magnetic resonance shifts for the $\mathrm{N}-\mathrm{CH}_{2}$ - protons in tris( $N, N$-diethyldithiocarbamato) iron(III) in a range of solvents and solvent mixtures we shall explore the effect of the solvent interactions on the two parameters, $\delta$, and the energy difference $\mathrm{E}$ where $\mathrm{E}={ }^{6} \mathrm{~A}_{1}-{ }^{2} \mathrm{~T}_{2}$, i.e., when $\mathrm{E}$ is positive the ground state is the ${ }^{2} \mathrm{~T}_{2}$ state and when $\mathrm{E}$ is negative the ground state is the ${ }^{6} \mathrm{~A}_{1}$ state.

In interpreting the NMR shift, $\Delta B$, obtained from the NMR spectra of paramagnetic molecules it is usual to consider two terms. First, the Fermi contact interaction where the contribution may be expressed ${ }^{7}$ as

$$
\Delta \mathrm{B}=\mathrm{a}\left\langle\mathrm{S}_{\mathrm{Z}}\right\rangle / \mathrm{g}_{\mathrm{N}} \mu_{\mathrm{N}}
$$

where $\left\langle\mathrm{S}_{z}\right\rangle$ is the time-averaged value of the $\mathrm{z}$ component of the electron spin and $\mathrm{a}$ is the hyperfine interaction constant measured in hertz. Secondly, we need to consider the dipolar term in the multipole expansion of the interaction of the electron orbital angular momentum and the electron spin dipolar-nuclear spin angular momentum. This may be expressed ${ }^{8}$ in terms of the magnetic moments components, $\mu^{2}$, and the magnetic susceptibility components, $\chi$, as

$$
\frac{\Delta B}{B}=-\frac{\mu_{B}^{2} \mu_{0}}{3 k T 4 \pi}\left\{\left[\mu_{\|}^{2}-\mu_{\perp}^{2}\right]\left(3 \cos ^{2} \theta-1\right)\right\} / 3 R^{3}
$$

where $\chi_{\|}=\frac{N \mu_{B}^{2}}{3 k T} \mu_{\|}^{2}$ and $\chi_{\perp}=\frac{N \mu_{B}^{2}}{3 k T} \mu_{\perp}^{2}$.
The magnetic moments are expressed in terms of the $\mathrm{E}$ and $\delta$-values in the appendix.

( $\mathrm{gN}_{\mathrm{N}}$ is the Lande g-value for the nucleus, $\mathrm{ge}_{\mathrm{e}}$ is the electron g-value $2.0023, \mu_{\mathrm{N}}$ the nuclear magneton, $\mu_{0}$ the permeability of a vacuum. The polar coordinates of the NMR nucleus in relation to the paramagnetic centre are $(\mathrm{R}, \theta, \phi)$, where the $z$-axis is the trigonal axis of the complex.)

Although equation (4) and equation (5) are approximations a combination of equation (4) and equation (5) will mirror rather closely the temperature dependence of the NMR shifts although care needs to be exercised in a detailed interpretation of the results. ${ }^{9}$

To proceed, therefore, we need to establish the form of equation (4) and equation (5) applicable to the analysis of the NMR study of the solvent interaction with the iron(III) dithiocarbamate.

A previous proton NMR study ${ }^{2}$ of tris $(N, N$-diethyldithiocarbamato) iron(III) indicated that the transition metal ion is in an intermediate crystal field environment such that the ${ }^{6} \mathrm{~A}_{1}$ state and the lowest lying ${ }^{2} T_{2}$ state for the $d^{5}$ ion are close in energy. Hence, we need to consider both states. We shall consider only the $\mathrm{Fe}^{3+}$ d-electrons interacting with the various protons within the ligands.

First, we shall examine the form of the NMR shift for the ${ }^{6} \mathrm{~A}_{1}$ state. Studies of iron(III) dithiocarbamate complexes with a ${ }^{6} \mathrm{~A}_{1}$ ground state well below the ${ }^{2} \mathrm{~T}_{2}$ state show that the NMR shift, $\Delta B$, is inversely proportional to the absolute temperature, $T$. This indicates that the origin of the NMR shift in this case arises through the contact term. ${ }^{10}$

For the ${ }^{2} \mathrm{~T}_{2}$ level we need to consider the contribution to the NMR shift by the term proportional to $\chi_{\|}-\chi_{\perp}$ and the Fenni interaction. Since in these compounds the effective crystal field environment of the iron atom is approximately of octahedral symmetry with a small most likely trigonal component $^{11.12}$ we shall assume that the form of the Fermi contact interaction is given in Golding et $a l^{3}$ The magnetic moments and the magnetic susceptibility components $\mathcal{X} \|$ and $\chi_{\perp}$ can be determined from the appropriate equations. ${ }^{13}$ For our case the appropriate equations are given in the appendix. Combining the above results yield the following.

$$
\frac{\Delta B}{B}=\alpha v_{2}+\beta y_{6}+\gamma\left(\chi_{\|}-\chi_{\perp}\right)
$$

where

$$
\begin{aligned}
& y_{2}=-\frac{(x+16 / 3) \exp (x)-(16 / 3) \exp (-x / 2)}{\exp (x)+2 \exp (-x / 2)+3 \exp (-E / k T)} \\
& y_{6}=-\frac{105 x \exp (-E / k T)}{\exp (x)+2 \exp (-x / 2)+3 \exp (-E / k T)} \\
& x=\zeta / k T
\end{aligned}
$$

The magnetic susceptibilities expressed as function of the distortion, $\delta$, spin-orbit coupling, $\zeta$, and temperature, $\mathrm{T}$, are given in the appendix.

We would expect the electronic and atomic structure of the complex at least near the transition metal ion not to be affected significantly by the solvent. Hence in analysing the 
NMR results we shall treat the solvent interaction as a perturbation where the dominant crystal field interaction wil] arise from the ligands. (As we shall show later the results confirm this approach.) Therefore, the parameters $\alpha, \beta$ and $\gamma$ in equation (6) would not change significantly for the $\mathrm{N}-\mathrm{CH}_{2}$ protons in tris $(N, N$-diethyldithiocarbamato) iron(III) from solvent to solvent and we shall treat these parameters as constant for a specific proton in an iron(III) dithiocarbamate. This is analogous to the method used to analyse the NMR shifts in a series of lanthanide complexes. ${ }^{15}$ Hence the temperature dependent NMR results for the $\mathrm{N}-\mathrm{CH}_{2}$ protons in tris( $N, N$-diethyldithiocarbamato) iron(III) in all solvents may be analysed as a single set of data using equation (6) where the energy separation of the ${ }^{6} \mathrm{~A}_{1}$ and the ${ }^{2} \mathrm{~T}_{2}$ states, $\mathrm{E}$, and the distortion parameter $\delta$ are allowed to vary from solvent to solvent. The best fit yields $\mathrm{E}$ and $\delta$ values for the system in each solvent with an overall set of values for the parameters $\alpha, \beta$ and $\gamma$.

\section{Results and Discussion}

The $60 \mathrm{MHz}$ proton NMR shift, $\Delta \mathrm{B}$, for the $\mathrm{N}-\mathrm{CH}_{2}$ protons in tris $(N, N$-diethyldithiocarbamato) iron(III) were determined in a number of solvents and in some solvent mixtures over a wide temperature range. (The diamagnetic contribution to the NMR shift was obtained by measuring the NMR shift of the $\mathrm{N}-\mathrm{CH}_{2}$ protons in the diamagnetic cobalt analogue.) In this paper six different solvents were used: acetone, benzene, carbon disulfide, chloroform; dimethylformamide and pyridine. Also three chlorofonndimethylformamide solvent mixtures were used: $0.75,0.49$ and 0.24 mole fraction of chloroform.

The $\Delta \mathrm{B}$ values for all the nine solvent systems were fitted to equation (6) where the variables $\mathrm{E}$ and $\delta$ were chosen for each solvent system to yield the best fit to all the data. The results are given in Table 1 .

Figure I illustrates the fit with the data for five solventschloroform, benzene, carbon disulfide, pyridine and dimethylformamide. (The dots indicate the experimental data and the

Table 1. The E and $\delta$ values, in $\mathrm{cm}^{-1}$, found to give the best fit to the experimental data using equation (6). The Q-values of Dance and Miller ${ }^{16}$ for some of the solvents are given

\begin{tabular}{|c|c|c|c|c|}
\hline solvent system & $\mathrm{E}$ & $\delta$ & $\mathrm{Q}_{1}$ & $\mathrm{Q}_{2}$ \\
\hline acetone & 499 & 74.0 & 4.65 & 0 \\
\hline benzene & 499 & 123.7 & 2.43 & 0 \\
\hline carbon disulfide & 510 & 80.7 & - & - \\
\hline chloroform & 426 & 274.3 & 2.91 & 1.81 \\
\hline dimethylformamide & 503 & 53.7 & 5.14 & 0 \\
\hline pyridine & 503 & 75.0 & 4.31 & 0 \\
\hline $\begin{array}{l}0.75 \text { mole fraction of chloroform } \\
\text { in dimethylformamide }\end{array}$ & 472 & 167.7 & - & - \\
\hline $\begin{array}{l}0.49 \text { mole fraction of chloroform } \\
\text { in dimethylformamide }\end{array}$ & 492 & 110.3 & - & - \\
\hline $\begin{array}{l}0.24 \text { mole fiaction of chloroform } \\
\text { in dimethylformamide }\end{array}$ & 496 & 81.7 & - & - \\
\hline
\end{tabular}

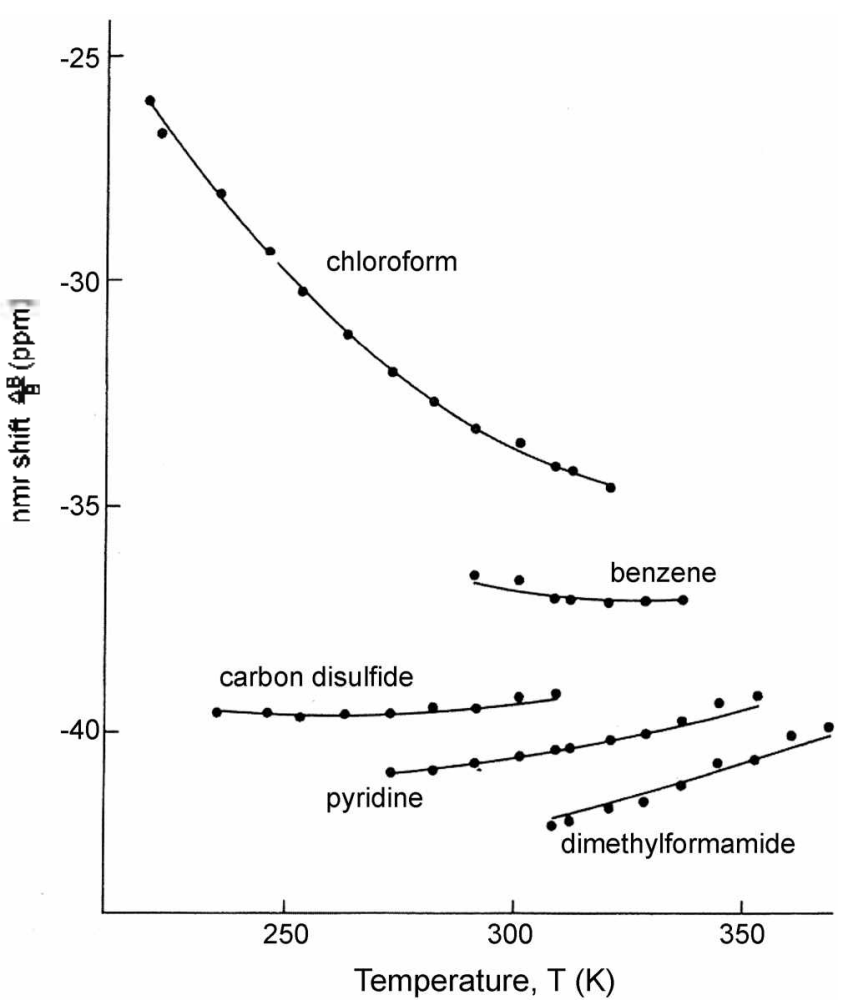

Figure 1. The temperature dependence of the NMR paramagnetic chemical shift of the $\mathrm{N}-\mathrm{CH}_{2}$ protons in tris $(N \mathrm{~N}$-diethyldithiocarbanato) iron(III) in a range of solvents.

curves the best fit of equation (6). The acetone results lie between the pyridine and the dimethylfomamide results).

The first significant factor about the results is that the $\mathbf{E}$ values are remarkably similar and correspond to a $\Delta$ value of about $27,500 \mathrm{~cm}^{-1}$. The variation in E corresponds to less than $0.4 \%$ variation in the octahedral symmetric component of the crystal fjeld potential. In addition the distortion parameter is small for all cases and hence not only is the trigonal crystal field component to the crystal field potential small but the variation from solvent to solvent is small. This supports our approach of treating the solvent interaction as a perturbation.

To illustrate the type of results we shall give the calculated NMR shifts for the chloroform case at five temperatures

Table 2. Calculated NMR shifts in the solvent chloroform when $\alpha$ $=5.784, \beta=3.235$ and $\gamma=-0.670$ with $\theta=0$. (a) Dipolar approximation when $\mathrm{R}=0.4 \mathrm{~nm}$. (b) Exact solution when $\mathrm{R}=0.4$ $\mathrm{nm}$. (c) Exact solution when $\mathrm{R}=0.432 \mathrm{~nm}$

\begin{tabular}{ccccc}
\hline $\begin{array}{c}\text { Temperature } \\
(\mathrm{K})\end{array}$ & $\begin{array}{c}\text { Observed } \\
\text { NMR shift } \\
(\mathrm{ppm})\end{array}$ & $\begin{array}{c}\text { Calculated } \\
\text { NMR shift } \\
(\mathrm{ppm}) \\
(\mathrm{a})\end{array}$ & $\begin{array}{c}\text { Calculated } \\
\text { NMR shift } \\
(\mathrm{ppm}) \\
(\mathrm{b})\end{array}$ & $\begin{array}{c}\text { Calculated } \\
\text { NMR shift } \\
(\mathrm{ppm}) \\
(\mathrm{c})\end{array}$ \\
\hline 225 & -26.9 & -27.3 & -21.9 & -27.6 \\
250 & -30.0 & -29.7 & -24.9 & -29.8 \\
275 & -32.1 & -31.8 & -27.6 & -31.7 \\
300 & -33.8 & -33.6 & -29.8 & -33.4 \\
325 & -34.6 & -35.0 & -31.7 & -34.8 \\
\hline
\end{tabular}


when $\alpha=5.784, \beta=3.235$ and $\gamma=-0.670$ and $\mathrm{R}=0.4 \mathrm{~nm}$ with $\theta=0$ using equation (6).

Using equation (6) with the magnetic susceptibility we have assumed the dipolar approximation. In doing so we do not need to know the R-value or the $\theta$-value as these will be absorbed into the $\gamma$-value as a factor that is temperature independent. On the other hand if we used the exact solution to replace the magnetic susceptibility term in equation (6) this in not the case. Furthermore, we would need to know the exact position of the proton NMR nucleus from the transition metal ion. In Table 2 we compare, first, the dipolar and the exact solutions between the calculated NMR shifts using the same $\alpha, \beta$ and $\gamma$-values, namely, columns (a) and (b). There is a marked difference highlighting the comments in the appendix. We may gain further insight into the dipolar approximation by adjusting $\mathrm{R}$ and $\theta$ to match the calculated NMR shifts given in column (a). If we choose $\mathrm{R}=0.432 \mathrm{~nm}$ in the exact calculation for the last term in equation (6) we obtain the results in column (c) arising from the electronproton interaction. If we choose $\mathrm{R}=0.34 \mathrm{~nm}$ and $\theta=0.1754$ $\pi$ the agreement is within $0.03 \mathrm{ppm}$. Thus we must acknowledge that care needs to exercised in using the last term in equation (6).

Dance and Miller, ${ }^{16}$ have developed an empirical parameterization of solute-solvent interactions, based on solvatochromic data from dipolar metal coordination complexes (including complexes with dithiolate ligands similar to the dithiocarbamates) and organic dyes, and including solvolysis activation free energy data. The solvent dependent observable energy is expressed as a sum of products of solute and solvent parameters $P_{i}$ and $Q_{i}$ respectively, which we may express for our case as

$$
\delta=\mathrm{P}_{0}+\mathrm{P}_{1} \mathrm{Q}_{1}+\mathrm{P}_{2} \mathrm{Q}_{2}
$$

where $Q_{1}$ is a measure of the electric field experienced by the solute in its solvent cavity and $Q_{2}$ is a measure of donor hydrogen bonding by the solvent. 225 data sparning 24 solvents and 16 solutes/phenomena were used in this parameterization. The Q-values of Dance and Miller ${ }^{16}$ for the appropriate solvents are given in Table 1.

A linear regression analysis of the $\delta$ values in Table 1 for the solvents with given $Q$ values yields

$$
\delta=184.4-24.87 \mathrm{Q}_{1}+89.70 \mathrm{Q}_{2}
$$

Hence from equation (8) with no solvent solute interactions $\left(\mathrm{Q}_{1}=\mathrm{Q}_{2}=0\right)$ we find that $\delta=184.4 \mathrm{~cm}^{-1}$. The contribution to $\delta$ from the second term in equation (8) is negative and ranges from about $-60 \mathrm{~cm}^{-1}$ to $-130 \mathrm{~cm}^{-1}$. The contribution to $\delta$ from $\mathrm{Q}_{2}$ is positive and is about $162.4 \mathrm{~cm}^{-1}$. This is consistent with X-ray studies ${ }^{17}$ which show that when iron(III) dithiocarbamates are crystallised from chloroform solution in at least some of the compounds chloroform molecules are hydrogen bonded to the sulfur atoms of the dithiocarbamate ligands.

Furthermore, the solvents used affect also the E-value. In this case a linear regression analysis of the $\mathrm{E}$ values in Table 1 for the solvents with given $Q$ values yields

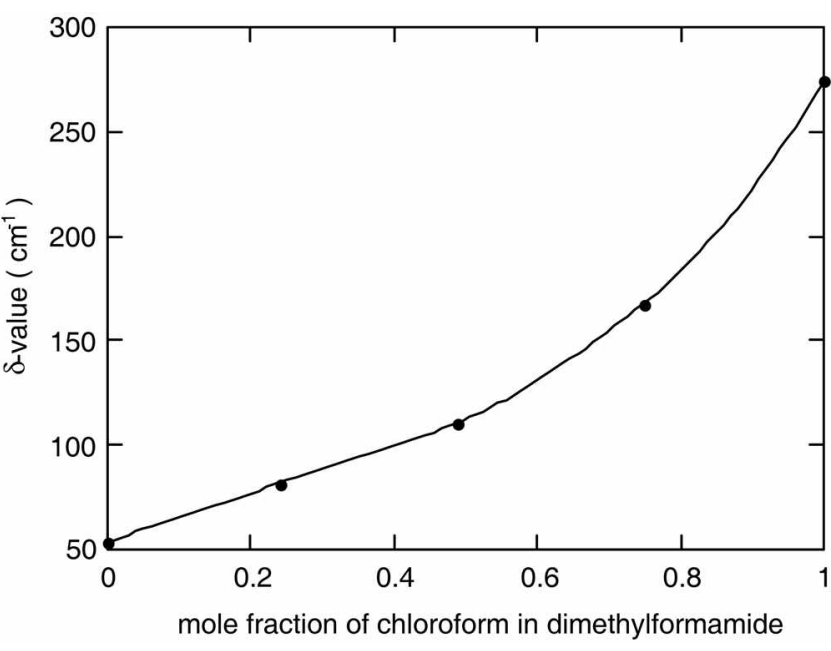

Figure 2. $\Lambda$ plot of the distortion parameter, $\delta$, as a function of the mole fraction of chloroform in dimethylformamide.

$$
\mathrm{E}=507.1-1.324 \mathrm{Q}_{1}-42.69 \mathrm{Q}_{2}
$$

This confirms that the E-value is also affected where the largest interaction is through the $Q_{2}$ term that for our solvents is specific to chloroform.

Finally, a plot of the distortion parameter, as a function of the mole fraction of chloroform in dimethylformamide clearly shows that there is not a linear relation between the mole fraction of chloroform and the distortion parameter.

As seen from Figure 2 the results imply that the dimethylformamide molecules at least partially shield the chloroform molecules from the iron(III) dithiocarbamate solute.

Furthermore, we may use (8) and (9) to estimate the Qvalues from the nmr determined $\mathrm{E}$ and $\delta$ values.

From Table 3 the $Q_{1}$ values for the mole fraction of chloroform in dimethylformamide form the simple relationship, $Q_{1}=5.08-2.29$ mole whereas $Q_{2}$ may be expressed as $Q_{2}=1.80$ mole $^{3}$. These equations reflect the very different nature of the $Q$-values.

In this analysis of the NMR data we have shown that the results for a variety of solvents and over a wide temperature range may be interpreted as arising from small changes in the crystal field environment of the iron atom due to two solvent interactions. One term is an intrinsic property of the solvent and the other term arises from a solute-solvent hydrogen bonding interaction. The model yields not only an

\begin{tabular}{|c|c|c|c|c|}
\hline solvent system & $\mathrm{E}$ & $\delta$ & $Q_{1}$ & $\mathrm{Q}_{2}$ \\
\hline carbon disulfide & 510 & 80.7 & 3.53 & 0 \\
\hline $\begin{array}{l}0.75 \text { mole fraction of chloroform } \\
\text { in dimethylformamide }\end{array}$ & 472 & 167.7 & 3.27 & 0.72 \\
\hline $\begin{array}{l}0.49 \text { mole fraction of chloroform } \\
\text { in dimethylformamide }\end{array}$ & 492 & 110.3 & 3.83 & 0.24 \\
\hline $\begin{array}{l}0.24 \text { mole fraction of chloroform } \\
\text { in dimethylformamide }\end{array}$ & 496 & 81.7 & 4.56 & 0.12 \\
\hline
\end{tabular}
explanation for the unusual NMR results but gives an insight

Table 3. The calculated Q-values from the E and $\delta$ values 
into the solvent-solute interactions in such systems. The outcome of our approach yields a new dimension in gaining an understanding of solvent interactions using NMR spectroscopy.

Finally, we should note that in our example the combination of the Fermi and dipolar electron-nuclear interactions given in (6) is a very good approximation since we treat our $\alpha, \beta$ and $\gamma$ values as unknown variables. As an example, we may treat the ratio $\left\{\left(3 \cos ^{2} \theta-1\right)\right\} / 3 R^{3}$ as an unknown factor associated with the $\gamma$-value. Thus the actual values of $\theta$ and $\mathrm{R}$ that we have used are unimportant. Hence in our analysis we do not require detailed knowledge of the three dimensional structure of the tris $(N, N$-diethyldithiocarbamato) iron(III) molecule. In summary, we have shown how (6) may be used under a range of conditions to interpret the numr shift of the $\mathrm{d}^{5}$-ion in a strong intermediate crystal field approximately of octahedral symmetry in a way that does not require knowledge of our $\mathrm{R}$-value nor the angle $\theta$. We vary the three terms in (6) to give the best fit, yielding realistic values for the $\zeta, \mathrm{E}$ and $\delta$ values. The three parameters $\alpha, \beta$ and $\gamma$ effectively compensate for the lack of detailed information of the electron-nuclear interactions.

\section{References}

1. Golding, R. M.; Kok, L. L.; Lehtonen, K.; Nigam, R. K. Atsst. J. Chem. 1975, 28, 1915 .

2. Golding, R. M.; Tennant, W. C.; Kaneka, C. R.; White, A. H. J. Chem. Phys. 1966, 45, 2688.

3. Golding, R. M.; Tennant, W. C.; Bailey, J. M. P.; Hudson, A. J. Chem. Phys. 1968, 48, 764.

4. Golding, R. M.; Lukeman, B. D.; Sinn, E. J. Chem. Phys. 1972, 56,4147 .

5. Golding, R. M. Pure Appl. Chem. 1972, 32, 123.

6. Perry, W. D.; Drago, R. S. J. Am. Chem. Soc. 1971, 03, 2183.

7. Golding R. M. Molec. Phys. 1964, 8,561.

8. Kurland, R. J.; McGarvey, B. R. J. Magh. Reson, 1970, 2, 286.

9. Golding, R. M.; Stubbs, L. C. J. Magn. Reson, 1979, 33, 627 .

10. Golding, R. M. Applied Wave Mechanics; Van Nostrand: London, 1969.

II. Golding, R. M.; Whitfield, H. J. Trans. Faraday Soc. 1966, 62, 1713 .

12. Golding, R. M. Molec, Phys. 1967, 12, 13.

13. Das, M.; Golding, R. M.; Livingston, S. E. Transition Met, Chem. $1978,3,112$.

14. Griffith, J. S. The Theory of Transition Metal Ions; Cambridge University Press: London, 1961.

15. Bergen, H. A.; Golding R. M. Aust. J. Chem, 1977, 30, 2361.

16. Miller, T. R., PhD thesis, Department of Chemistry, University of Wisconsin: Madison, Wisconsin, USA, 1973.

17. Raston, C. L.; White, A. H. J. Chem. Soc. 1975, 2405.

18. Golding, R. M.; Pascual, R. O.; Vrancich, J. Molec. Phys. 1976, $73 \mathrm{I}$.

\section{Appendix}

Interpreting the NMR chemical shif for paramagnetic systems requires, first, an understanding of the electron-nuclear interactions and, secondly, how to use that knowledge to extract meaningful information from the data available. Normally, we have to make an intuitive guess of the possible electronic structure and then compare the observed with the experimental NMR shift. In the case of the iron (III) dithiocarbamates the $\mathrm{d}^{5}$-ion is in a crystal field environment close to octahedral symmetry. Hence, to a good approximation the electron-nuclear interaction may be represented by the Fermi contact interaction in an intermediate crystal field of octahedral symmetry as given by Golding et $a l^{3}$ Neglecting configurational mixing of the excited states the Fermi contact terms for the ${ }^{2} \mathrm{~T}_{2}$ and ${ }^{\circ} \mathrm{A}_{1}$ states may be expressed proportional to $y_{2}$ and $y_{0}$ as in equation (6). Next we need to include a further term arising from the electron-nuclear interaction, the pseudo contact tenm. In general the pseudo contact term is a complex function in terms of the co-ordinate system describing the atoms associated with the electron and the nuclear spin. Specifically, in terms of the NMR nucleus the electron-bearing atom is expressed as $(\mathrm{R}, \theta$, $\phi)$. When $\mathrm{R}$ is non-zero, the complex function may be expressed in terms of $I / R "$. The term for $\mathrm{t} / \mathrm{R}^{3}$ is known as the dipolar term. As $\mathrm{R} \rightarrow \infty$ the complex function tends to the dipolar value. For more detail see Golding et al. ${ }^{\text {I8 }}$ (The constant factor in the exponential term for the d-electron radial function was chosen as $2.2 / a_{0}$ where $a_{0}$ is the Bohr radius.)

Furthermore, the dipolar term may be expressed in terms of the magnetic susceptibilities. 'Thus we may express for a $\mathrm{d}^{5}$-ion in an intermediate crystal field with a small distortion from octahedral symmetry the total NMR paramagnetic chemical shif from the electron-rnclear interaction by three terms as given by equation ( 6 ).

The NMR shif from the magnetic moments, $\mu_{\alpha}^{2}$, where $\alpha$ is $\|$ or $\perp$ may be expressed as follows:

$\mu i_{\alpha}^{2}=\frac{\chi_{1} \exp \left(-E_{1} / k T\right)+\chi_{2} \exp \left(-E_{2} / k T\right)+\chi_{3} \exp \left(-E_{3} / k T\right)}{\exp \left(-E_{1} / k T\right)+\exp \left(-E_{2} / k T\right)+\exp \left(-E_{3} / k T\right)+\exp (-E / k T)}$

where

$E_{1}=-\frac{\zeta}{4}+\frac{\delta}{6}+\frac{X}{2} ; E_{2}=-\frac{\zeta}{4}+\frac{\delta}{6}-\frac{X}{2} ;$

$E_{3}=\frac{\zeta}{2}-\frac{\delta}{3} ; X=\left\{\frac{9}{4} \xi+\zeta \delta+\delta^{2}\right\}^{1 / 2}$

$E$ is the energy difference between the ${ }^{2} \mathrm{~T}_{2}$ and ${ }^{\circ} \mathrm{A}_{1}$ states. $E$ is positive when the ${ }^{2} \mathrm{~T}_{2}$ is the ground state.

Furthermore,

$$
\begin{aligned}
& \chi_{1}=3 H_{11}^{2}-6\left\{\frac{H_{12}^{2}}{\left(E_{1}-E_{2}\right)}+\frac{H_{13}^{2}}{\left(E_{1}-E_{3}\right)}\right\} k T \\
& \chi_{2}=3 H_{22}^{2}-6\left\{\frac{H_{12}^{2}}{\left(E_{2}-E_{1}\right)}+\frac{H_{33}^{2}}{\left(E_{2}-E_{3}\right)}\right\} k T \\
& \chi_{3}=3 H_{33}^{2}-6\left\{\frac{H_{13}^{2}}{\left(E_{3}-E_{1}\right)}+\frac{H_{23}^{2}}{\left(E_{3}-E_{2}\right)}\right\} k T
\end{aligned}
$$

For $\mu \hat{\mu}_{\perp}^{2}$

$H_{11}=\frac{g_{e}}{2} A^{2}+\sqrt{2} A B ; H_{22}=\frac{g_{e}}{2} B^{2}-\sqrt{2} A B ; H_{33}=0 ;$

$H_{12}=-\frac{g_{z}}{2} A B+\frac{1}{\sqrt{2}}\left(A^{2}-B^{2}\right) ; H_{13}=-\frac{g_{g}}{2} B-\frac{\mathrm{l}}{\sqrt{2}} A ; H_{23}=-\frac{g_{g}}{2} A+\frac{1}{\sqrt{2}} B$

For $\mu \mu_{\|}^{2}$

$H_{11}=\frac{g_{2}}{2}\left(B^{2}-A^{2}\right)+B^{2} ; H_{22}=\frac{g_{2}}{2}\left(A^{2}-B^{2}\right)+A^{2} ; H_{33}=\frac{g_{2}}{2}-1 ;$

$H_{12}=g_{e} A B+A B ; H_{13}=0 ; H_{23}=0$

where

$A=\left\{\frac{1}{2}+\frac{1}{2}\left(\frac{\zeta}{2}+\delta\right) X^{-1}\right\}^{1 / 2} ; B=-\left\{\frac{1}{2}-\frac{1}{2}\left(\frac{\zeta}{2}+\delta\right) X^{-1}\right\}^{12}$ 